\title{
NeW Results on the Windy Postman Problem
}

\author{
A. Corberán ${ }^{1 *}$ I. Plana ${ }^{1}$, G. Reinelt ${ }^{3}$, J.M. Sanchis ${ }^{2}$ and D. Theis ${ }^{3}$ \\ ${ }^{1}$ Dept. d'Estadística i Investigació Operativa, Universitat de València, Spain \\ 2 Dept. de Matemática Aplicada, Universidad Politécnica de Valencia, Spain \\ ${ }^{3}$ Dept. of Computer Science, University of Heidelberg, Germany
}

June 20, 2007

\begin{abstract}
In this paper we study the Windy Postman Problem. This is a well known Arc Routing Problem which contains the Mixed Chinese Postman Problem as a special case. We present here a full description of the polyhedron associated to the Windy Postman Problem over graphs with up to 4 vertices and 10 edges. We introduce two new families of facet-inducing inequalities and we prove that these inequalities and the already known Odd Zigzag inequalities are mod-2 cut inequalities. Moreover, a Branch \& Cut (B\&C) algorithm that incorporates two new separation algorithms for all the previously mentioned inequalities and a new heuristic procedure to obtain upper bounds are presented. Finally, the performance of the B\&C algorithm over several sets of large WPP and MCPP instances, with up to 3000 nodes and 9000 links, shows that up to our knowledge this is the best algorithm for the exact resolution of the WPP and the MCPP.
\end{abstract}

Key Words: Polyhedral Combinatorics, Facets, Arc Routing, Windy Postman Problem, Mixed Postman Problem.

\section{Introduction}

Arc Routing Problems consist of finding one or several shortest closed walks (tours) on the links of a given graph $G$ satisfying certain conditions. Graph $G$ can be undirected (all the links are edges that can be traversed, at the same cost, in both directions), directed (all the links are arcs that must be traversed in a given direction), mixed (having edges and arcs simultaneously) and windy (an undirected graph with two costs associated with each edge representing the cost of traversing it in each direction). Obviously, routing problems defined on windy graphs generalize the corresponding problems defined on undirected, directed and mixed graphs.

In this paper we deal with the Windy Postman Problem, WPP. This problem can be defined as follows. Given an undirected and connected graph $G=(V, E)$ with two non-negative costs $c_{i j}$ and $c_{j i}$ associated with each edge $(i, j) \in E$ corresponding to the cost of traversing it from $i$ to $j$ and from $j$ to $i$, respectively, find the tour on $G$ traversing each edge at least once, at minimum cost. This problem was first introduced by Minieka (1979), is an NP-hard problem (Guan 1984 and Brucker 1981) which can be solved in polynomial time if $G$ is Eulerian (Win, 1987).

*corresponding author: angel.corberan@uv.es 
Win (1987) and Grötschel \& Win (1992) proposed a cutting plane algorithm for the WPP based on a previous polyhedral study that, as far as we know, is the first polyhedral approach that has been applied to the resolution of a NP-hard Arc Routing Problem. They proved that the Odd-cut inequalities and the $k$-wheel inequalities are facet-inducing, although only the separation of the first ones was implemented in their cutting plane algorithm. More recently, Corberán, Plana \& Sanchis (2006) present a new family of facet-inducing inequalities for the Windy General Routing Problem, the Odd Zigzag inequalities, which also applies to the WPP. These inequalities generalize the 3-wheel inequalities proposed by Win (1987) for the WPP.

On the other hand, the WPP contains the mixed version of the well-known Chinese Postman Problem, MCPP, as a special case. As the WPP, this NP-hard problem (Papadimitriou, 1976) can be handled with a formulation using two variables associated with each edge (see Christofides et al., 1984, and Ralphs, 1993) and therefore it can be considered as a WPP in which some variables have infinite cost. Hence, the results presented here for the WPP can be easily applied to the MCPP.

In the next section we define the problem, introduce the notation that will be used in this paper and present some known results. Section 3 is devoted to fully describe the polyhedra associated with the WPP over graphs with up to 4 vertices and 10 edges. Here we also present two new families of facet-inducing inequalities, the Even-Even and Odd-Odd Zigzag inequalities. We prove that these inequalities and the Odd Zigzag inequalities are mod-2 cut inequalities in Section 4. The Branch \& Cut algorithm for the resolution of the WPP is described in section 5. The computational experiments performed on a wide set of WPP and MCPP large instances are described in Section 6, while Section 7 presents the conclusions.

\section{Problem definition and known results}

As mentioned in the Introduction, the Windy Postman Problem consists of finding a minimum cost closed walk traversing all the edges of a windy graph $G=(V, E)$ (WPP tour) at least once.

Given a node subset, $S \subseteq V$, let $\delta(S)$ denote the edge set with an end-point in $S$ and the other in $V \backslash S$ and let $E(S)$ be the set of edges with both end-points in $S$. Given two node subsets $S_{1}, S_{2} \subseteq V,\left(S_{1}, S_{2}\right)$ will represent the set of edges with one end-point in $S_{1}$ and the other in $S_{2}$. A vertex is called even (odd) if it is incident with an even (odd) number of edges. A subset $S \subset V$ is called even (odd) if it contains an even (odd) number of odd vertices.

Let $x_{i j}$ be the number of times edge $(i, j)$ is traversed from $i$ to $j$ in a WPP tour. Given $F \subseteq E$, we denote by $x(F)=\sum_{\{i, j\} \in F}\left(x_{i j}+x_{j i}\right)$, and given $\left(S_{1}, S_{2}\right)$, we denote by $x\left(S_{1}: S_{2}\right)$ the sum of the variables associated with the traversal from $S_{1}$ to $S_{2}$ of the edges in $\left(S_{1}, S_{2}\right)$,

$$
x\left(S_{1}: S_{2}\right)=\sum_{i \in S_{1}, j \in S_{2}} x_{i j}
$$

Note that $x\left(S_{1}, S_{2}\right)=x\left(S_{1}: S_{2}\right)+x\left(S_{2}: S_{1}\right)$. The formulation given by Win (1987) and by Grötschel \& Win (1992) is: 


$$
\text { Minimize } \quad \sum_{(i, j) \in E}\left(c_{i j} x_{i j}+c_{j i} x_{j i}\right)
$$

s.t.:

$$
\begin{aligned}
x_{i j}+x_{j i} & \geq 1, \quad \forall(i, j) \in E \\
\sum_{(i, j) \in \delta(i)}\left(x_{i j}-x_{j i}\right) & =0, \quad \forall i \in V \\
x_{i j}, x_{j i} & \geq 0 \\
x_{i j}, x_{j i} \text { integer } &
\end{aligned}
$$

where conditions conditions (1) imply that each edge will be traversed at least once and conditions (2) force the (directed) graph represented by the tour to be symmetric. The above system includes an equation associated with each vertex. The $|V|$ equations (2) will be referred to as the system equations and any $|V|-1$ of them are linearly independent.

Let $\operatorname{WPP}(G)$ be the convex hull of all the tours $x \in \mathbb{Z}^{2|E|}$ satisfying (1) to (4). In Grötschel \& Win (1988) it is shown that $\operatorname{WPP}(G)$ is an unbounded polyhedron with dimension $2|E|-|V|+1$, and that the following inequalities are, under mild conditions, facet-inducing:

- Trivial inequalities (3)

- Traversing inequalities (1)

- The Odd-cut inequalities:

$$
x(\delta(S)) \geq|\delta(S)|+1, \quad \forall S \subset V, \quad|\delta(S)| \text { is odd }
$$

- The $k$-wheel inequalities.

Inequalities (5) are equivalent to the following ones, which contain fewer non-zero elements:

$$
x(S: V \backslash S) \geq \frac{|\delta(S)|+1}{2}, \quad \forall S \subset V, \quad|\delta(S)| \text { is odd }
$$

More recently, Corberán, Plana \& Sanchis (2006) present a new family of facet-inducing inequalities for the Windy General Routing Problem, the Odd Zigzag inequalities, which also applies to the WPP. These inequalities generalize the 3 -wheel inequalities.

In Corberán, Plana \& Sanchis (2005) it is also shown that all the facet-inducing inequalities for $\operatorname{WPP}(G)$ except (3) and (1) are weak configuration inequalities. Associated with a weak configuration inequality, we have a configuration graph, $G_{\mathcal{C}}=(\mathcal{B}, \mathcal{E})$, where node set $\mathcal{B}$ is a partition of $V, \mathcal{B}=\left\{B_{1}, B_{2}, \ldots, B_{r}\right\}$, and $\mathcal{E}$ contains an edge $\left(B_{i}, B_{j}\right)$ for each edge $(u, v)$ of $G$ with $u \in B_{i}, v \in B_{j}$. In other words, $G_{\mathcal{C}}$ is the graph resulting after shrinking node sets $B_{i}$, $i=1, \ldots, r$, into a single vertex each, but keeping all the edges. This concept is similar to that of configuration inequalities by Naddef \& Rinaldi (1991). They only differ in the fact that, in a configuration inequality, all the variables $x_{u v}$ associated with the edges $\{u, v\} \in\left(B_{i}, B_{j}\right)$ have equal coefficients in the inequality, while a weak configuration inequality can have variables $x_{u v}$, $x_{s t}$, with $u, s \in B_{i}$ and $v, t \in B_{j}$, with different coefficients. This is the case of the Odd Zigzag inequalities and the inequalities presented later in this paper. In Corberán, Plana \& Sanchis (2005) is also included a 'lifting' theorem that states that if a weak configuration inequality is facet-inducing for $\operatorname{WPP}\left(G_{\mathcal{C}}\right)$, it is also facet-inducing for $\operatorname{WPP}(G)$.

Given that we present in this paper a separation algorithm for the Odd zigzag inequalities and that they are related to the new inequalities presented below, we describe them briefly in the context of the WPP. 


\subsection{Odd Zigzag inequalities}

These inequalities cut off fractional solutions containing a zigzag associated with variables with 0.5 value such as the one shown in Figure 1a. Consider a partition of the set of vertices $V$ into 4 parts, $M^{1}, M^{2}, M^{3}$ and $M^{4}$, where each $M^{i}$ contains an odd number of odd vertices. Let $\alpha_{i j}$ denote the number of edges in $\left(M^{i}, M^{j}\right)$. Let us call $\mathcal{H}=\left(M^{1}, M^{2}\right) \cup\left(M^{3}, M^{4}\right)$ (horizontal edges) and $\mathcal{D}=\left(M^{2}, M^{3}\right) \cup\left(M^{1}, M^{4}\right)$ (diagonal edges). Note that $\mathcal{H} \cup \mathcal{D}=\delta\left(M^{1} \cup M^{3}\right)$. Let us define a subset of required edges $\mathcal{F} \subset(\mathcal{H} \cup \mathcal{D})$ satisfying (see Figure 1b, where edges in $\mathcal{F}$ are represented in bold lines)

$$
|\mathcal{H} \backslash \mathcal{F}|+|\mathcal{D} \cap \mathcal{F}|=|\mathcal{D} \backslash \mathcal{F}|+|\mathcal{H} \cap \mathcal{F}|
$$

The configuration graph $G_{\mathcal{C}}$ associated with the Odd Zigzag inequalities is defined by the partition of $V$ and the set $\mathcal{F}$ above, and by the following pair of coefficients associated with each edge $e_{i j}$ (see Figure $1 \mathrm{~b}$ ):

$$
\left(c_{i j}, c_{j i}\right)=\left\{\begin{array}{lll}
(0,2) & \forall e_{i j} \in \mathcal{H} \backslash \mathcal{F}, \quad i \in M^{1} \cup M^{3}, \quad j \in M^{2} \cup M^{4} \\
(2,2) & \forall e_{i j} \in \mathcal{H} \cap \mathcal{F} \\
(1,3) & \forall e_{i j} \in \mathcal{D} \cap \mathcal{F}, \quad i \in M^{1} \cup M^{3}, \quad j \in M^{2} \cup M^{4} \\
(1,1) & \text { otherwise }
\end{array}\right.
$$

The corresponding Odd Zigzag inequality is then

$$
x\left(\delta\left(M^{1} \cup M^{2}\right)\right)+2 x\left(M^{2}: M^{1}\right)+2 x\left(M^{4}: M^{3}\right)+2 x\left(F_{z z}\right) \geq \alpha_{13}+\alpha_{24}+\alpha_{14}+\alpha_{23}+2|\mathcal{H} \cap \mathcal{F}|+2
$$

where $x\left(F_{z z}\right)$ denotes the variables associated with the edges in $\mathcal{F}$ in the direction given by the zigzag, i.e. in the direction $M^{1}-M^{2}-M^{3}-M^{4}-M^{1}$. Set $\mathcal{F}$ can be understood in the following way. The edges in $G_{\mathcal{C}}$ except two given edges $e_{13} \in\left(M^{1}, M^{3}\right)$ and $e_{24} \in\left(M^{2}, M^{4}\right)$ can be oriented to obtain a (directed) symmetric graph. Given any such orientation, set $\mathcal{F}$ is defined by all the required edges that have been oriented in the opposite direction to the zigzag. In particular, set $\mathcal{F}$ in Figure $1 \mathrm{~b}$ is defined from the orientation associated with the fractional solution shown in Figure 1a. Note that such a set $\mathcal{F}$ satisfies the condition (7). Other sets $\mathcal{F}$ can be defined to obtain valid inequalities but only the one shown in Figure 1b has an associated inequality violated by the fractional solution in Figure 1a.

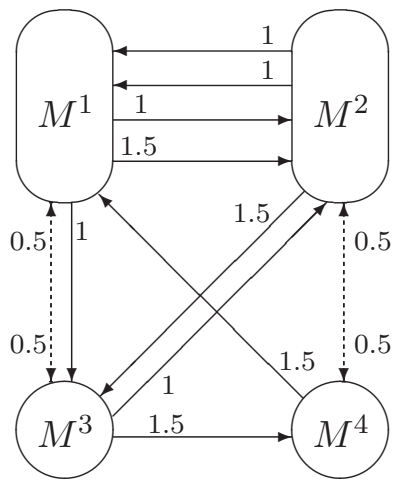

(a)

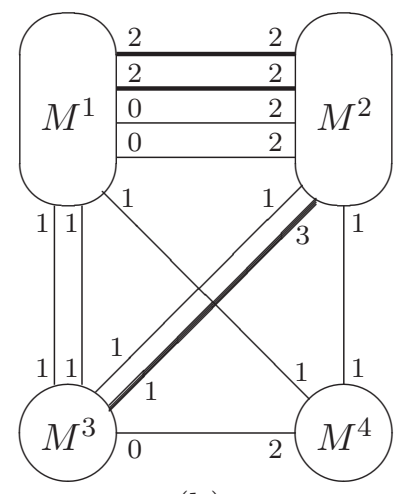

(b)

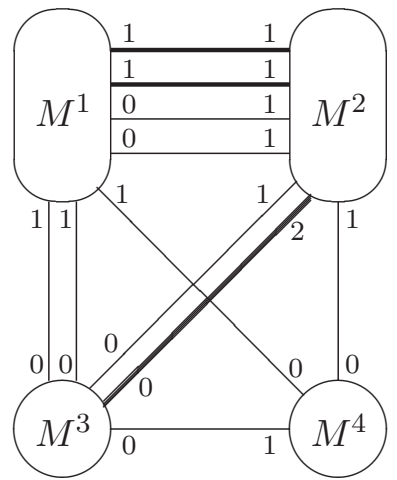

(c)

Figure 1: A fractional solution and an Odd Zigzag configuration with set $\mathcal{F}$ in bold lines.

As it occurs with Odd-cut inequalities, Odd Zigzag inequalities can be written also in sparse form. To illustrate this, consider the inequality (8) represented in Figure 1b. Due to the 
symmetry equation associated with node $M_{1}$, the term $x\left(V \backslash M_{1}: M_{1}\right)$ in the inequality can be replaced by $x\left(M_{1}: V \backslash M_{1}\right)$ to obtain an equivalent inequality. Proceeding in a similar way with node $M_{2}$ and then dividing by 2 , we obtain the following equivalent inequality, whose coefficients are shown in Figure 1c:

$$
x\left(M^{1} \cup M^{2}: M^{3} \cup M^{4}\right)+x\left(M^{2}: M^{1}\right)+x\left(M^{4}: M^{3}\right)+x\left(F_{z z}\right) \geq \frac{1}{2}\left(\alpha_{13}+\alpha_{24}+\alpha_{14}+\alpha_{23}\right)+|\mathcal{H} \cap \mathcal{F}|+1
$$

\section{Small WPP polyhedra and new Zigzag inequalities}

When we were designing the separating algorithm for the Odd Zigzag inequalities we generated some fractional solutions which satisfy all the Odd-cut inequalities and we represented them to find violated inequalities visually. We look for directed paths formed by four edges whose associated variables have a 1.5 value joining 4 odd nodes (such as that in Figure 1a). We realized that some fractional solutions have four edges with 1.5 value but the path they formed is not a directed path (one or two edges are traversed in the opposite direction to the others) and it joins two odd nodes and two even nodes (such as those in Figures 4a, 4b, 5a and 6a). Given that these fractional solutions can not be separated by an Odd Zigzag inequality, some other classes of facet-inducing inequalties must exist. In order to find them, we have made a full description of the polyhedra associated with the WPP defined on graphs with 4 vertices and up to 10 edges.

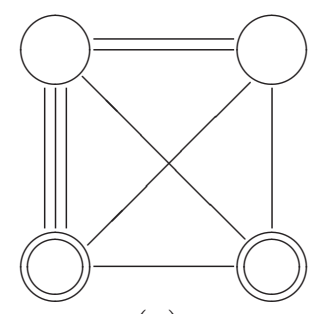

(a)

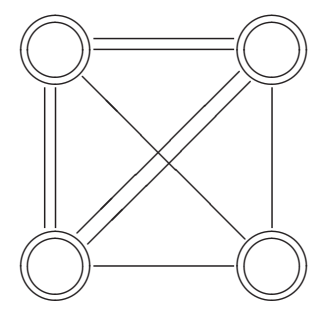

(d)

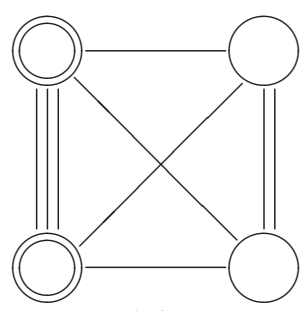

(b)

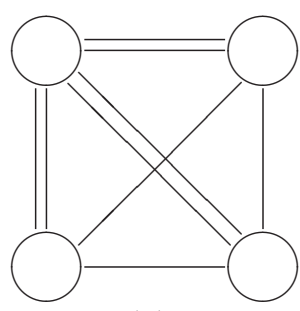

(e)

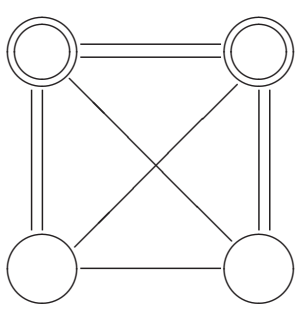

(c)

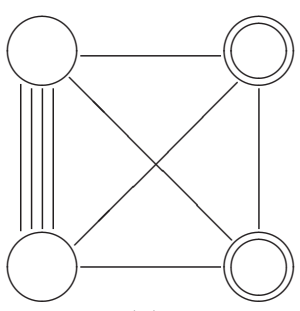

(f)

Figure 2: Graphs with 4 nodes and 9 edges

We first considered the complete graph with 6 edges. Then we added edges to obtain all the possible graphs with 7,8,9 and 10 edges, respectively. For each graph, the full description of its WPP associated polyhedron was obtained using PORTA (Christof, Jünger \& Reinelt, 1991). Trivial and traversing facets appear in the description of all the polyhedra and we do not mention them in what follows. Graphs with 7 edges are fully described with Odd-cut inequalities, while graphs with 6 and 8 edges are described with Odd-cut and Odd Zigzag inequalities. All different graphs with 9 edges are depicted in Figure 2, where odd degree nodes are represented by a double circle. The polyhedra associated with graphs in Figures $2 \mathrm{a}, 2 \mathrm{~b}$ and $2 \mathrm{c}$ are fully described with Odd-cut inequalities, while for graph in Figure 2d Odd-cut and Odd Zigzag inequalities are 
needed and for graph in Figure 2e no more inequalities are needed. However, the polyhedron associated with graph in Figure $2 \mathrm{f}$ (and Figure 3a) contains new facet-defining inequalities that do not correspond to any of the classes previously mentioned.

There are 10 different graphs with 4 vertices and 10 edges. Two of them have only even degree vertices and their corresponding polyhedra are completely described with trivial and traversing inequalities. Three other graphs need Odd-cut inequalities for their polyhedra to be described while four other graphs also need Odd Zigzag inequalities. The polyhedron associated with the remaining graph, shown in Figure 3b, contains new facet-defining inequalities.

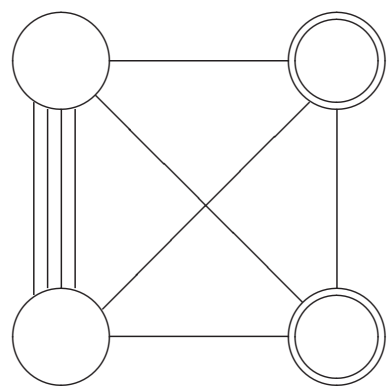

(a)

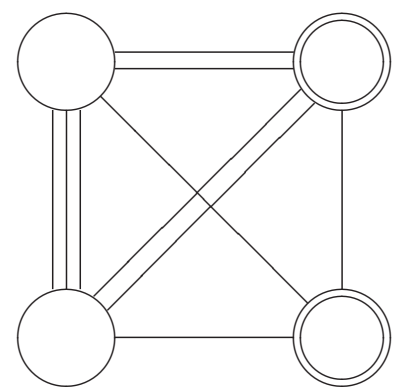

(b)

Figure 3: WPP instances whose polyhedra have new facet-inducing inequalities.

Hence we have found facet-inducing inequalities different from Odd-cut and Odd Zigzag inequalities only for the instances shown in Figure 3. For the instance shown in Figure 3a, the two fractional solutions in Figure 4 can be obtained. Two similar fractional solutions can also be found for the instance depicted in Figure 3b. Note that in Figure $4 \mathrm{~b}$ the arrangement of the nodes has been changed in order to better illustrate the inequalities that will be described in the following sections. One of the two variables associated with each one of the edges $(1,2)$, $(2,3),(3,4)$ and $(4,1)$ have 1.5 value in these solutions. In the solution shown in Figure 4a these edges are traversed in the directions $(1,2),(2,3),(4,3)$ and $(1,4)$ while in the solution depicted in Figure 4b they are traversed in the directions $(1,2),(2,3),(3,4)$ and $(1,4)$. Each of these two solutions violates the corresponding inequality described in what follows. Each type of inequality is determined by the situation of the two odd degree nodes and by the traversal of the 4 edges $(1,2),(2,3),(3,4)$ and $(4,1)$. Note that the four edges with 1.5 value do not form a directed path. In the first case there are two edges that are traversed in the opposite direction to the others whilst in the second case there is only one. For the sake of simplicity, for each type of inequality we will say that an edge is oriented 'in the direction of the zigzag' if it is traversed as described above.

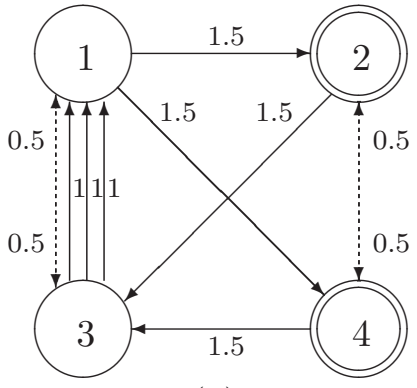

(a)

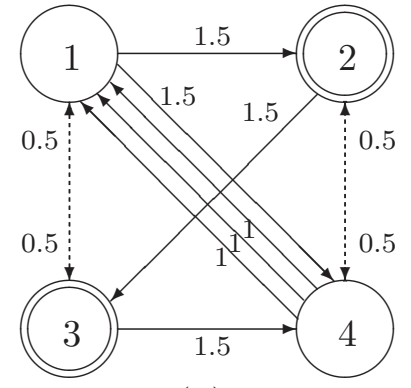

(b)

Figure 4: Fractional solutions 


\subsection{Even-Even Zigzag inequalities}

The inequalities presented here cut fractional solutions similar to those represented in Figures $4 \mathrm{a}$ and $5 \mathrm{a}$. The name of the inequalities refers to the degree of the two shores of the edge cutset $\delta\left(M^{1} \cup M^{3}\right)$. Note that, in this case, the number of edges in this cutset is even.

Consider a partition of the set of vertices $V$ into 4 parts, $M^{1}, M^{2}, M^{3}$ and $M^{4}$, where $M^{2}$ and $M^{4}$ contains an odd number of odd vertices while $M^{1}$ and $M^{3}$ contains an even number of odd vertices. Let us define a subset of edges $\mathcal{F} \subset \delta\left(M^{1} \cup M^{3}\right)$ satisfying:

$$
\left|\left(M^{1}, M^{2} \cup M^{4}\right) \backslash \mathcal{F}\right|+\left|\left(M^{2} \cup M^{4}, M^{3}\right) \cap \mathcal{F}\right|=\left|\left(M^{1}, M^{2} \cup M^{4}\right) \cap \mathcal{F}\right|+\left|\left(M^{2} \cup M^{4}, M^{3}\right) \backslash \mathcal{F}\right|
$$

(see Figure 5b, where edges in $\mathcal{F}$ are represented in bold lines)

The configuration graph $G_{\mathcal{C}}$ associated with the Even-Even Zigzag inequalities is defined by the partition of $V$ and the set $\mathcal{F}$ above, and by the following pair of coefficients associated with each edge $e_{i j}$ (see Figure $5 \mathrm{~b}$ ):

$$
\left(c_{i j}, c_{j i}\right)=\left\{\begin{array}{lll}
(0,2) & \forall e_{i j} \in\left(M^{1}, M^{2} \cup M^{4}\right) \backslash \mathcal{F}, \quad i \in M^{1}, \quad j \in M^{2} \cup M^{4} \\
(2,2) & \forall e_{i j} \in\left(M^{1}, M^{2} \cup M^{4}\right) \cap \mathcal{F}, \quad \forall e_{i j} \in\left(M^{2}, M^{4}\right) \\
(1,3) & \forall e_{i j} \in\left(M^{3}, M^{2} \cup M^{4}\right) \cap \mathcal{F}, \quad i \in M^{3}, \quad j \in M^{2} \cup M^{4} \\
(1,1) & \text { otherwise }
\end{array}\right.
$$

Note that the pairs with different coefficients correspond to the edges in the left hand side of (10). The corresponding Even-Even Zigzag inequality is then

$$
\begin{gathered}
x\left(\delta\left(M^{3}\right)\right)+2 x\left(M^{2}, M^{4}\right)+2 x\left(M^{2}: M^{1}\right)+2 x\left(M^{4}: M^{1}\right)+2 x\left(F_{z z}\right) \geq \\
\geq \alpha_{13}+2 \alpha_{24}+\alpha_{23}+\alpha_{34}+2\left|\delta\left(M^{1}\right) \cap \mathcal{F}\right|+2
\end{gathered}
$$

where $x\left(F_{z z}\right)$ denotes the variables associated with the edges in $\mathcal{F}$ in the direction of the zigzag, i.e. $\left(M^{1} \rightarrow M^{2}\right),\left(M^{2} \rightarrow M^{3}\right),\left(M^{4} \rightarrow M^{3}\right)$ and $\left(M^{1} \rightarrow M^{4}\right)$.

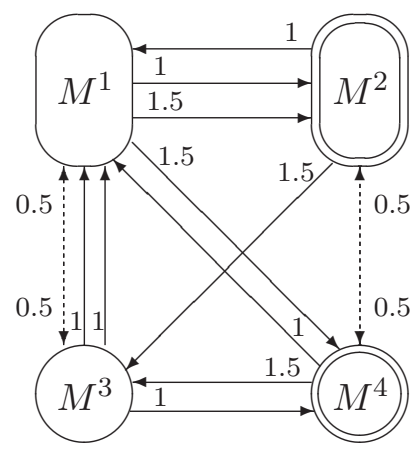

(a)

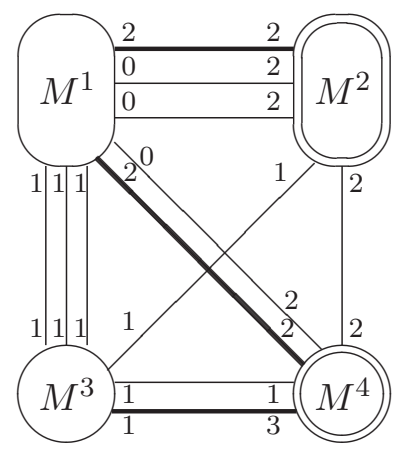

(b)

Figure 5: A fractional solution and an Even-Even Zigzag configuration with set $\mathcal{F}$ in bold lines.

Set $\mathcal{F}$ can be chosen in the following way. The edges in $G_{\mathcal{C}}$ except two given edges $e_{13}, f_{13} \in$ $\left(M^{1}, M^{3}\right)$ and a given edge $e_{24} \in\left(M^{2}, M^{4}\right)$ can be oriented to obtain a (directed) symmetric graph. Given any such orientation, set $\mathcal{F}$ is defined by all the edges that have been oriented in the opposite direction to the zigzag. In particular, set $\mathcal{F}$ in Figure $5 \mathrm{a}$ is defined from the orientation associated with the fractional solution. Note that such a set $\mathcal{F}$ satisfies the condition (10). 
Theorem 1 Even-Even Zigzag inequalities (11) are valid for $W P P(G)$.

Proof: Let $F(x) \geq c_{0}$ denote the inequality and let $x$ be a WPP tour. Given that each WPP tour must traverse every edge in one of its two possible directions, $x$ has at least an $F$-cost of $c_{0}-2$. We can suppose that $x$ traverses all the edges in the left hand side of (10) in the direction corresponding to their lower coefficient, i.e., from $M^{1} \cup M^{3}$ to $M^{2} \cup M^{4}$ (otherwise we have an extra F-cost of two and we are done). Let $K$ be the number of such edges. Since the nodes $M^{2}$ and $M^{4}$ are odd, the tour $x$ cannot traverse each edge exactly once and an extra matching on these two nodes is needed. Notice that all these matchings have $F$-cost of at least 2 (and hence we are done) except the matching with $F$-cost zero defined by two edges not in $\mathcal{F}$ traversed from $M^{1}$ to $M^{2}$ and from $M^{1}$ to $M^{4}$, respectively. So we can suppose that $x$ traverses at least $K+2$ times from $M^{1} \cup M^{3}$ to $M^{2} \cup M^{4}$ and, hence, it traverses also $K+2$ times from $M^{2} \cup M^{4}$ to $M^{1} \cup M^{3}$. Given that the number of edges in the right hand side of (10) is $K, x$ traverses at least 2 extra times from $M^{2} \cup M^{4}$ to $M^{1} \cup M^{3}$, with an $F$-cost of at least 2 .

Theorem 2 Even-Even Zigzag inequalities (11) are facet-inducing for $W P P(G)$ if $G_{\mathcal{C}} \backslash \mathcal{F}$ is a complete graph, there are three edges $e_{13}, f_{13} \in\left(M^{1}, M^{3}\right)$ and $e_{24} \in\left(M^{2}, M^{4}\right)$ and the remaining edges in $G_{\mathcal{C}}$ can be oriented to induce a (directed) symmetric graph where all the edges in $\delta\left(M^{1} \cup\right.$ $\left.M^{3}\right) \backslash \mathcal{F}$ are oriented in the direction of the zigzag and all the edges in $\mathcal{F}$ are oriented in the opposite direction.

Proof: We will first prove that the inequalities are facet-inducing for $\operatorname{WPP}\left(G_{\mathcal{C}}\right)$. We need to find twice the number of edges in $G_{\mathcal{C}}$ minus 3 linearly independent WPP tours satisfying $F(x)=c_{0}$. Each tour is a vector with two components $x_{i j}, x_{j i}$ associated with each edge $e=\{i, j\}$ in $G_{\mathcal{C}}$.

We first select the six components corresponding to the given edges $e_{13}, f_{13}$ and $e_{24}$. Let $x^{d}$ denote the incidence vector of the symmetric subgraph induced by the orientation mentioned in the theorem. Note that the six selected components in $x^{d}$ are zero and that $F\left(x^{d}\right)=c_{0}-6$.

Since $G_{C} \backslash \mathcal{F}$ is a complete graph, it is possible to select another 4 components associated with 4 edges not in $\mathcal{F}, e_{12} \in\left(M^{1}, M^{2}\right), e_{14} \in\left(M^{1}, M^{4}\right), e_{23} \in\left(M^{2}, M^{3}\right)$ and $e_{43} \in\left(M^{4}, M^{3}\right)$, in the direction given by the zigzag. It can be seen that, for each unselected variable $x_{i j}$, a tour based on vector $x^{d}$ can be constructed satisfying $F(x)=c_{0}$ and such that it uses the variable $x_{i j}$ once more than $x^{d}$ plus some of the selected components. Moreover, we can build 7 more WPP linearly independent tours from $x^{d}$ by adding to it only some of the selected components, also satisfying $F(x)=c_{0}$. If we subtract $x^{d}$ from all the tours and arrange them in rows, we obtain a full-rank matrix. Hence the Even-Even Zigzag inequalities are facet-inducing for $\operatorname{WPP}\left(G_{\mathcal{C}}\right)$. By applying the lifting theorem in Corberán, Plana \& Sanchis (2005), they are also facet-inducing for $\operatorname{WPP}(G)$.

Also Even-Even Zigzag inequalities can be written in sparse form. Due to the symmetry equation associated with node $M^{3}$, the term $x\left(V \backslash M^{3}: M^{3}\right)$ in the inequality can be replaced by $x\left(M^{3}: V \backslash M^{3}\right)$ to obtain an equivalent inequality that when divided by two has the form:

$$
\begin{aligned}
x\left(M^{3}: V \backslash\right. & \left.M^{3}\right)+x\left(M^{2}, M^{4}\right)+x\left(M^{2}: M^{1}\right)+x\left(M^{4}: M^{1}\right)+x\left(F_{z z}\right) \geq \\
& \geq \frac{1}{2}\left(\alpha_{13}+2 \alpha_{24}+\alpha_{23}+\alpha_{34}\right)+\left|\delta\left(M^{1}\right) \cap \mathcal{F}\right|+1
\end{aligned}
$$




\subsection{Odd-Odd Zigzag inequalities}

In this section we present another family of inequalities that cut fractional solutions like those shown in Figures 4b and 6a. Again, the name of the inequalities refers to the degree of the two shores of the edge cutet $\delta\left(M^{1} \cup M^{3}\right)$. In this case, the number of edges in this cutset is odd.

Consider a partition of the set of vertices $V$ into 4 parts, $M^{1}, M^{2}, M^{3}$ and $M^{4}$, where $M^{2}$ and $M^{3}$ contain an odd number of odd vertices while $M^{1}$ and $M^{4}$ contain an even number of odd vertices. Note that $\delta\left(M^{1} \cup M^{3}\right)$ contains an odd number of edges. Let us define a subset of edges $\mathcal{F} \subset \delta\left(M^{1} \cup M^{3}\right)$ satisfying:

$$
\begin{gathered}
\left|\left(M^{1}, M^{2} \cup M^{4}\right) \backslash \mathcal{F}\right|+\left|\left(M^{3}, M^{4}\right) \backslash \mathcal{F}\right|+\left|\left(M^{2}, M^{3}\right) \cap \mathcal{F}\right|+1= \\
\left|\left(M^{1}, M^{2} \cup M^{4}\right) \cap \mathcal{F}\right|+\left|\left(M^{3}, M^{4}\right) \cap \mathcal{F}\right|+\left|\left(M^{2}, M^{3}\right) \backslash \mathcal{F}\right|
\end{gathered}
$$

(see Figure 6b, where edges in $\mathcal{F}$ are represented in bold lines)

The configuration graph $G_{\mathcal{C}}$ associated with the Odd-Odd Zigzag inequalities is defined by the partition of $V$ and the set $\mathcal{F}$ above, and by the following pair of coefficients associated with each edge $e_{i j}$ (see Figure 6b):

$$
\left(c_{i j}, c_{j i}\right)= \begin{cases}(0,1) & \forall e_{i j} \in\left(M^{1}, M^{2} \cup M^{4}\right) \backslash \mathcal{F}, \quad i \in M^{1}, \quad j \in M^{2} \cup M^{4} \\ (0,1) & \forall e_{i j} \in\left(M^{3}, M^{4}\right) \backslash \mathcal{F}, \quad i \in M^{3}, \quad j \in M^{4} \\ (2,1) & \forall e_{i j} \in\left(M^{2}, M^{3}\right) \cap \mathcal{F}, \quad i \in M^{2}, \quad j \in M^{3} \\ (1,1) & \text { otherwise }\end{cases}
$$

Again, the pairs with different coefficients correspond to the edges in the left hand side of (13). The corresponding Odd-Odd Zigzag inequality is then

$$
\begin{gathered}
x\left(M^{2} \cup M^{4}: M^{1} \cup M^{3}\right)+x\left(M^{1}, M^{3}\right)+x\left(M^{2}, M^{4}\right)+x\left(M^{3}: M^{2}\right)+x\left(F_{z z}\right) \geq \\
\geq \alpha_{13}+\alpha_{24}+\left|\left(M^{2}, M^{3}\right) \backslash \mathcal{F}\right|+|\mathcal{F}|+1
\end{gathered}
$$

where $x\left(F_{z z}\right)$ denotes the variables associated with the edges in $\mathcal{F}$ in the direction of the zigzag, i.e. $\left(M^{1}, M^{2}\right),\left(M^{2}, M^{3}\right),\left(M^{3}, M^{4}\right)$ and $\left(M^{1}, M^{4}\right)$.

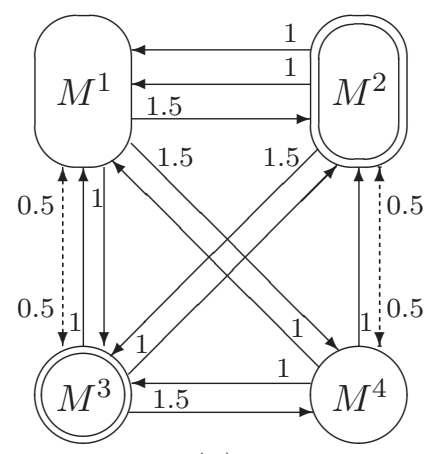

(a)

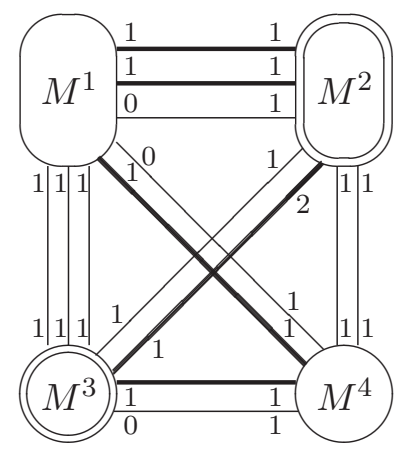

(b)

Figure 6: A fractional solution and an Odd-Odd Zigzag configuration with set $\mathcal{F}$ in bold lines.

Theorem 3 Odd-Odd Zigzag inequalities (14) are valid for $W P P(G)$. 
Proof: Let $F(x) \geq c_{0}$ denote the inequality and let $x$ be a WPP tour. Given that each WPP tour must traverse every edge in one of its two possible directions, $x$ has at least an $F$-cost of $c_{0}-1$. We can suppose that $x$ traverses all the edges in the left hand side of (13) in the direction corresponding to their lower coefficient, i.e., from $M^{1} \cup M^{3}$ to $M^{2} \cup M^{4}$ (otherwise we have an extra F-cost of 1 and we are done). Let $K$ be the number of such edges. Since the nodes $M^{2}$ and $M^{3}$ are odd, the tour $x$ cannot traverse each edge exactly once and an extra matching on these two nodes is needed. Notice that all these matchings have $F$-cost of at least 1 (and hence we are done) except the matching with $F$-cost zero defined by 3 edges not in $\mathcal{F}$ traversed from $M^{1}$ to $M^{2}$, from $M^{1}$ to $M^{4}$ and from $M^{3}$ to $M^{4}$, respectively. So we can suppose that $x$ traverses at least $K+3$ times from $M^{1} \cup M^{3}$ to $M^{2} \cup M^{4}$ and, hence, it traverses also $K+3$ times from $M^{2} \cup M^{4}$ to $M^{1} \cup M^{3}$. Given that the number of edges in the right hand side of (13) is $K+1$, $x$ traverses at least 2 extra times from $M^{2} \cup M^{4}$ to $M^{1} \cup M^{3}$, with an $F$-cost of at least 2 .

Theorem 4 Odd-Odd Zigzag inequalities (14) are facet-inducing for $W P P(G)$ if $G_{\mathcal{C}} \backslash \mathcal{F}$ is a complete graph, there are three edges $e_{13} \in\left(M^{1}, M^{3}\right), e_{24} \in\left(M^{2}, M^{4}\right)$ and $e_{14} \in\left(M^{1}, M^{4}\right) \cap \mathcal{F}$ and the remaining edges in $G_{\mathcal{C}}$ can be oriented to induce a (directed) symmetric graph where all the edges in $\delta\left(M^{1} \cup M^{3}\right) \backslash \mathcal{F}$ are oriented in the direction of the zigzag and all the edges in $\mathcal{F}$ are oriented in the opposite direction.

Proof: The proof is similar to that of Theorem 2 and is omitted here.

\section{Zigzag inequalities and mod-2 cuts}

Let $\min \{c x \mid A x \leq b, \quad x$ integer $\}$ be an Integer Program with $A$ and $b$ integer. Many combinatorial optimization problems can be written in this form. In the WPP case, the system $A x \leq b$ consists of the symmetry equations, traversing, Odd-cut and trivial inequalities. For $k$ integer, a mod- $k$ cut based on the system $A x \leq b$ of valid inequalities has the form $u^{T} A x \leq\left\lfloor u^{T} b\right\rfloor$ where the vector $u$ has components in the set $\left\{0, \frac{1}{k}, \ldots, \frac{k-1}{k}\right\}$ and $u^{T} A$ is integer.

In this section we will prove that all the previously described Zigzag inequalities are mod-2 cuts. Let us begin with Odd Zigzag inequalities. Add the following inequalities multiplied by $\frac{1}{2}$ :

- the Odd-cut inequality associated with $M^{1}: \quad x\left(M^{1}: V \backslash M^{1}\right) \geq \frac{1}{2}\left(\alpha_{12}+\alpha_{14}+\alpha_{13}+1\right)$,

- the Odd-cut inequality associated with $V \backslash M^{3}: \quad x\left(V \backslash M^{3}: M^{3}\right) \geq \frac{1}{2}\left(\alpha_{13}+\alpha_{23}+\alpha_{34}+1\right)$,

- the traversing inequalities corresponding to the edges in $\left(M^{2}, M^{4}\right): x_{i j}+x_{j i} \geq 1, \forall(i, j) \in$ $\left(M^{2}, M^{4}\right)$

- the traversing inequalities corresponding to the edges in $\mathcal{F}: \quad x_{i j}+x_{j i} \geq 1, \forall(i, j) \in \mathcal{F}$

- the trivial inequalities corresponding to the variables associated with the edges in $\mathcal{F}$ in the direction given by the zigzag,

- the trivial inequalities corresponding to the variables associated with the edges in $\left(M^{1} \cup\right.$ $\left.M^{3}, M^{2} \cup M^{4}\right) \backslash \mathcal{F}$ in the opposite direction to the zigzag, and

- the symmetry equation corresponding to $M^{2}: \quad x\left(M^{2}: V \backslash M^{2}\right)-x\left(V \backslash M^{2}: M^{2}\right)=0$ 
We obtain an inequality with all its coefficients integer while its RHS is:

$$
\frac{1}{2}\left(\frac{1}{2}\left(\alpha_{12}+\alpha_{14}+\alpha_{13}+1\right)+\frac{1}{2}\left(\alpha_{13}+\alpha_{23}+\alpha_{34}+1\right)+\alpha_{24}+|\mathcal{F}|\right) .
$$

From condition (7), it can be seen that this RHS is equal to

$$
\frac{1}{2}\left(\alpha_{13}+\alpha_{24}+\alpha_{14}+\alpha_{23}+2|H \cap F|+1\right),
$$

and since the LHS is integer and $\alpha_{13}+\alpha_{24}+\alpha_{14}+\alpha_{23}$ is an even number, the RHS can be rounded up to

$$
\frac{1}{2}\left(\alpha_{13}+\alpha_{24}+\alpha_{14}+\alpha_{23}+2|H \cap F|+2\right),
$$

which is exactly the Odd Zigzag inequality in sparse form (9).

To obtain the Even-Even Zigzag inequalities in sparse form (12), we add the following inequalities multiplied by $\frac{1}{2}$ : the Odd-cut inequalities associated with $M^{2}$ and with $M^{4}$, the traversing inequalities associated with edges in $\left(M^{1}, M^{3}\right),\left(M^{2}, M^{4}\right)$ and $F$, the trivial inequalities corresponding to the variables associated with the edges in $\mathcal{F}$ in the direction given by the zigzag (here, $\left(M^{1} \rightarrow M^{2}\right),\left(M^{2} \rightarrow M^{3}\right),\left(M^{4} \rightarrow M^{3}\right)$ and $\left.\left(M^{1} \rightarrow M^{4}\right)\right)$, the trivial inequalities corresponding to the variables associated with the edges in $\left(M^{1} \cup M^{3}, M^{2} \cup M^{4}\right) \backslash F$ in the opposite direction to the zigzag and the symmetry equation corresponding to set $M^{3}$ and then we round up the RHS.

Finally, the Odd-Odd Zigzag inequality (14) is obtained by adding the following inequalities multiplied by $\frac{1}{2}$ : the Odd-cut inequalities associated with $M^{2}$, with $V \backslash M^{3}$ and with set $M^{3} \cup M^{4}$, the traversing inequalities associated with edges in $\left(M^{1}, M^{3}\right),\left(M^{2}, M^{4}\right)$ and $F$, the trivial inequalities corresponding to the variables associated with the edges in $\mathcal{F}$ in the direction given by the zigzag (here, $\left(M^{1} \rightarrow M^{2}\right),\left(M^{2} \rightarrow M^{3}\right),\left(M^{3} \rightarrow M^{4}\right)$ and $\left.\left(M^{1} \rightarrow M^{4}\right)\right)$ and the trivial inequalities corresponding to the variables associated with the edges in $\left(M^{1} \cup M^{3}, M^{2} \cup M^{4}\right) \backslash F$ in the opposite direction to the zigzag.

\section{A Branch \& Cut for the WPP}

We have implemented a Branch \& Cut algorithm for the WPP in which, besides the well known heuristic and exact procedures for separating violated Odd-cut inequalities (see for instance Corberán, Plana \& Sanchis, 2007), new separation algorithms for identifying Odd, Even-Even and Odd-Odd Zigzag inequalities are incorporated. Moreover, a polynomial time algorithm for identifying maximally violated mod-2 cuts has been added as well as a heuristic algorithm producing feasible solutions from the LP fractional solutions.

\subsection{Zigzag Separation procedure 1}

This algorithm is designed to separate fractional solutions $x^{*}$ similar to those shown in Figures 1a, 5a and 6a. For the sake of simplicity we suppose here that most of the $x^{*}$ components are integer except some pairs with values $(1.5,0)$ and some pairs with values $(0.5,0.5)$. Figure 7 shows a directed graph associated with such a fractional solution in which the number beside each $\operatorname{arc}(i, j)$ denotes the value of its corresponding variable $x_{i j}^{*}$.

We are looking for a zigzag configuration such that its corresponding inequality is violated by $x^{*}$. Zigzag inequalities associated with a configuration with an edge $(i, j)$ linking different 


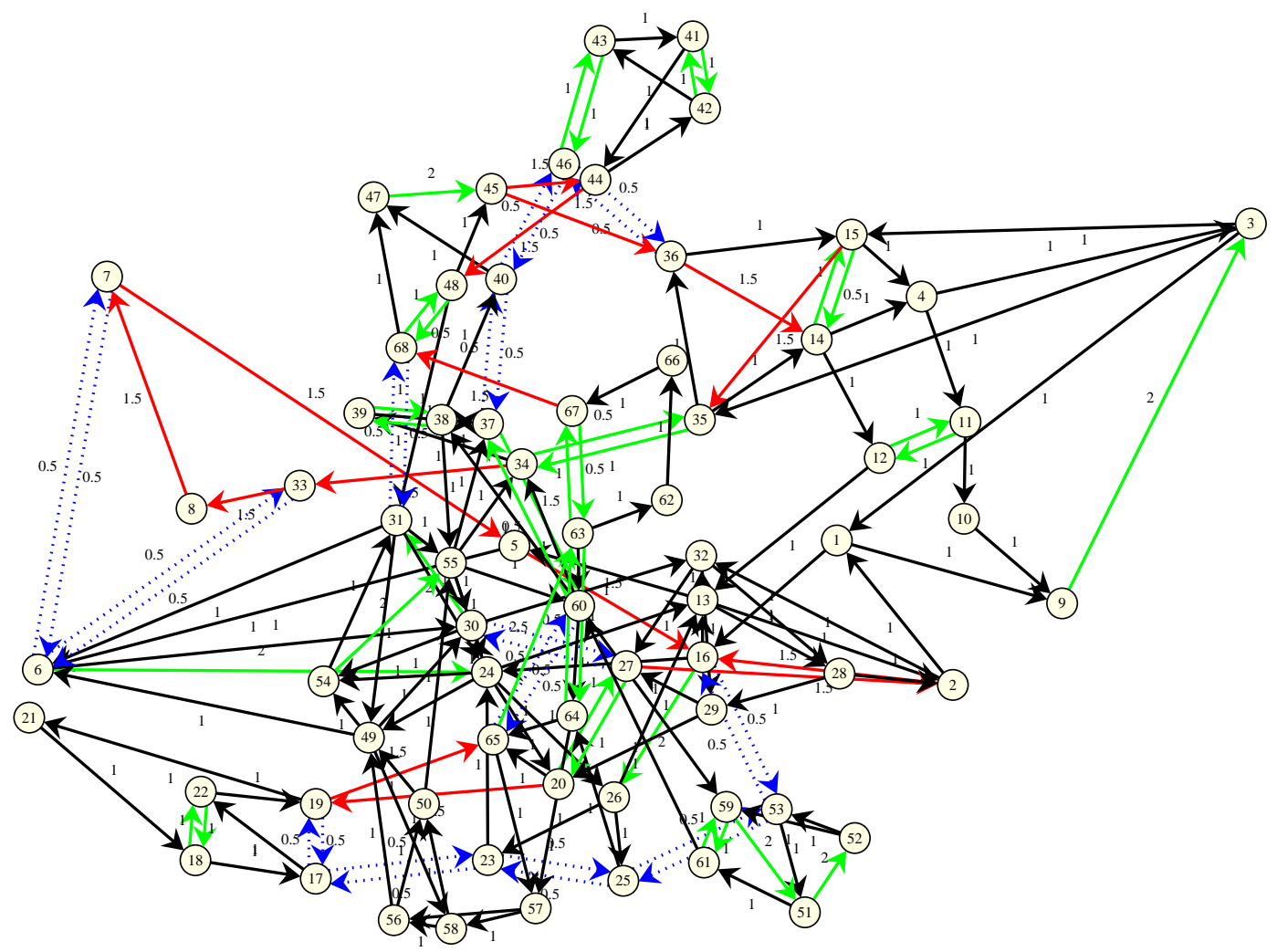

Figure 7: Fractional solution.

sets $M^{k}$ and satisfying $x_{i j}^{*}+x_{j i}^{*} \geq 2$ does not seem to be violated by $x^{*}$. Hence, each of these edges $(i, j)$ must belong to one of the sets $M^{k}$ and nodes $i$ and $j$ are shrunk in a first step. Moreover, edges $(i, j)$ traversed exactly once, i.e., satisfying $x_{i j}^{*}=1$ and $x_{j i}^{*}=0$ or viceversa, do not seem to affect the violation of the inequality and therefore they are deleted. All the resulting isolated nodes are also removed.

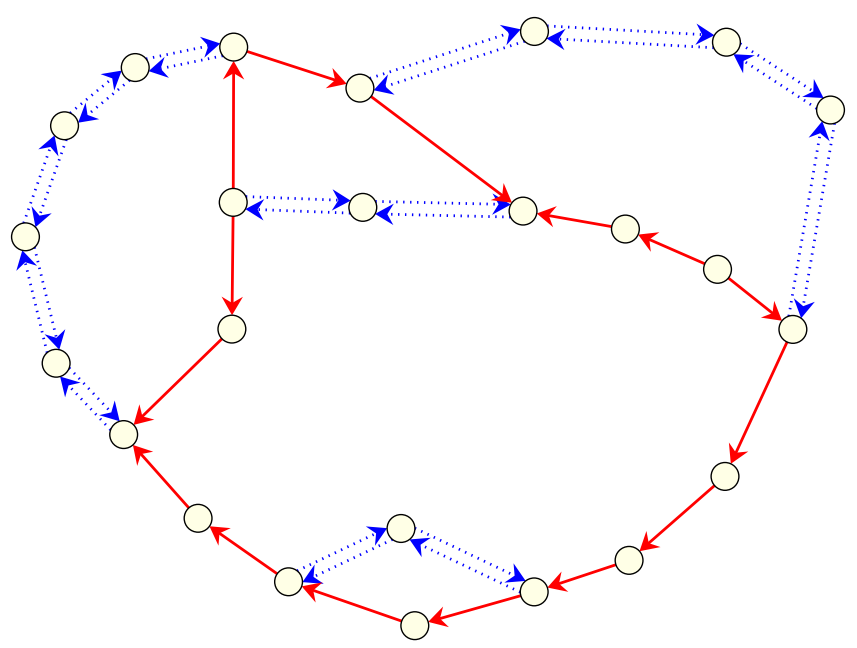

Figure 8: Shrunk fractional solution. 
At this point we should have one (maybe several) subgraph as the one depicted in Figure 8 containing only the edges $(i, j)$ satisfying $\left(x_{i j}^{*}, x_{j i}^{*}\right)=(1.5,0)$, depicted as arcs in solid lines, or $\left(x_{i j}^{*}, x_{j i}^{*}\right)=(0.5,0.5)$ represented by two opposite arcs in dotted lines. For each of these subgraphs we proceed as follows. All nodes incident with just two edges are iteratively shrunk into one of its adjacent nodes. Then we also shrink all the pairs of nodes linked by two parallel edges. Figure 9a shows the graph obtained after applying this procedure to the one in Figure 8.

Then we have a cycle formed by edges with values $(1.5,0)$ whose nodes are linked by an edge with values $(0.5,0.5)$, which we call chords (see Figure 9a). Note that each chord divides the cycle into two parts. We iteratively select two chords and label their end-nodes as seeds (since they are the seeds for sets $M^{k}$ ). We check if these two chords cross each other, i.e., each of the two halves defined by a chord contains exactly one of the seeds associated with the other chord. If so, the remaining chords are studied. For each one of these chords, if one of the two halves defined by it contains 3 seeds then we shrink the other half (which contains just one seed). If this process can be done for all the chords, we obtain a graph like the one shown in Figure $9 \mathrm{~b}$, which corresponds to a zigzag configuration. However, if a chord defines halves with two seeds each, the procedure fails and we proceed to select another pair of chords. Finally, the zigzag configuration on the original graph is built and its corresponding inequality is checked for violation by $x^{*}$.

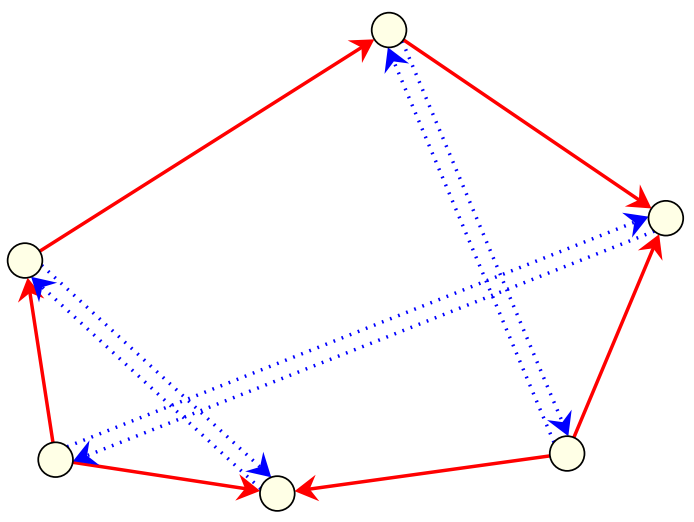

(a)

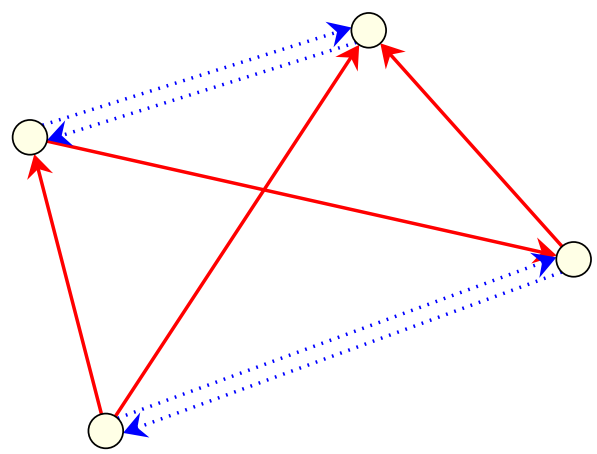

(b)

Figure 9: Cycle with chords and an Odd-Odd Zigzag configuration.

Since Zigzag inequalities can also be violated by solutions $x^{*}$ having fractional values different from 1.5 and 0.5 , the above procedure is applied to any LP fractional solution as follows:

(1) edges $(i, j)$ such that $x_{i j}^{*}+x_{j i}^{*}>1$ are shrunk, except those described in (2)

(2) edges $(i, j)$ such that $1.25<x_{i j}^{*}+x_{j i}^{*}<1.75$ and $x_{i j}^{*}<0.2$ or $x_{j i}^{*}<0.2$ are handled as satisfying $\left(x_{i j}^{*}, x_{j i}^{*}\right)=(1.5,0)$ or viceversa.

(3) for the remaining edges, $x_{i j}^{*}+x_{j i}^{*}=1$, those such that $x_{j i}^{*}<0.1$ are considered as satisfying $\left(x_{i j}^{*}, x_{j i}^{*}\right)=(1,0)$ and are deleted and the ones such that $0.1<x_{i j}^{*}<0.4$ or $0.1<x_{j i}^{*}<0.4$ are shrunk.

(4) all the other edges are handled as satisfying $\left(x_{i j}^{*}, x_{j i}^{*}\right)=(0.5,0.5)$. 


\subsection{Zigzag Separation procedure 2}

Unlike the previous algorithm, which is capable of finding Odd, Even-Even and Odd-Odd violated Zigzag inequalities, the procedure described in this section is designed to separate only Odd Zigzag inequalities. The idea of the algorithm is that the fractional solutions similar to the one shown in Figure 1a satisfy the Odd-cut inequalities corresponding to the (odd) sets $M^{k}$, $k=1,2,3,4$, as an equality and it works as follows.

During the execution of the Branch \& Cut algorithm all the Odd-cut inequalities found are stored. The separation procedure considers all the stored inequalities which are satisfied by $x^{*}$ as an equality. Then we select the shores $S \subset V$ of these inequalities such that all the pairs of variables associated with edges in $(S, V \backslash S)$ have values $(1,0)$ except the variables associated with 3 edges which have values $(1.5,0),(0,1.5)$ and $(0.5,0.5)$ respectively. Three such sets are iteratively selected. If they have no nodes in common, these 3 sets and the rest of $V$ are the candidates to be the sets $M^{k}$ of the Odd-Zigzag configuration. Finally, if the sets can be arranged in such a way that the arcs defined by the variables with value 1.5 form a directed cycle, then we have an Odd-Zigzag configuration, whose associated inequality is checked for violation by $x^{*}$.

\subsection{Mod-2 cuts separation procedure}

The previous separation algorithms are heuristics procedures and they can fail in finding violated Zigzag inequalities. In this section we use the polynomial algorithm presented in Caprara, Fischetti and Letchford (2000) for the separation of maximally violated mod- $k$ cuts. Since we have proved that all the Zigzag inequalities are mod-2 cuts, it is likely that some of the most violated mod-2 cuts are Zigzag inequalities which, as they are facet inducing, can be useful for the $\mathrm{B} \& \mathrm{C}$ algorithm.

Let $A x \leq b$ be a system of valid inequalities. Let us suppose that we find an integer vector $u \geq 0$ such that $u^{T} A$ is even while $u^{T} b$ is odd. For every feasible WPP tour $x, A x \leq b$ is satisfied and also $u^{T} A x \leq u^{T} b=2 s+1$ for an integer $s$. Given that $u^{T} A x$ is an even number, the inequality $u^{T} A x \leq 2 s$ is also valid. As any fractional solution $x^{*}$ satisfies $u^{T} A x^{*} \leq u^{T} b=2 s+1$, then inequality $u^{T} A x \leq 2 s$ can maximally be violated by 1 . This maximal violation is achieved if $u^{T} A x^{*}=u^{T} b$, that is, if $u_{i}=0$ for all $i$ such that $A_{i} x^{*}<b_{i}$. Dividing by two we obtain the inequality

$$
\frac{1}{2} u^{T} A x^{*} \leq s
$$

which is valid, has all its coefficients integer and its maximal violation is $\frac{1}{2}$. We look for such mod-2 cuts.

Let $x^{*}$ be a fractional solution and let $A x \leq b$ be a system of valid inequalities such that $A x^{*}=b$. In our case, this system is formed by all the binding inequalities of the last LP plus an inequality for each symmetry equality. We have to find a $0-1$ vector $u$ such that $u^{T} A$ is even and $u^{T} b$ is odd. Then we have to solve the following system of congruences:

$$
A^{T} u \equiv 0 \quad(\bmod 2) \quad \text { and } \quad b^{T} u \equiv 1(\bmod 2)
$$

Each solution $u$ of (16) yields a maximally violated mod-2 cut. Usually, if there is a cut, then there are many. Let us suppose that, after applying Gaussian elimination to system (16) there are a number $f$ of free variables. Given the values for these variables can be chosen in $\{0,1\}$, we have $2^{f}$ different solutions for (16), although the corresponding mod-2 cuts may not be different. A nice description of different strategies to select the cuts that are added to the LP can be found in Wenger (2004). We have used the procedure implemented by Fricke and Oswald (2006) to 
solve (16). When a solution with free variables is obtained, we take the cut resulting from setting all free variables to 0 and the cuts resulting from setting exactly one of the free variables to 1 and the remaining ones to 0 , up to a maximum of 50 cuts.

Given that the previous procedure is computationally expensive and that we have faster separation algorithms for Zigzag and Odd-cut inequalities (which are also mod-2 cuts), we call this routine only before branching at the root node.

\subsection{Initial relaxation and cutting plane algorithm}

The initial LP relaxation contains the Odd-cut inequalities associated with the odd degree vertices and with the connected components of the subgraph of $G$ induced by the odd degree vertices, if $|S|$ is odd. At each iteration of the cutting plane algorithm the separation procedures are called in the following order:

1. Odd cut separation heuristics.

2. Exact Odd-cut separation.

3. When no violated Odd-cut inequalities are found, run the Zigzag Separation procedures 1 and 2 .

4. At the end of the root node, if an optimal solution has not been found, the mod-2 cuts separation procedure is executed before branching.

The cutting plane procedure is applied at each node of the tree until no new violated inequalities are found or a stopping criterium, called tailing-off, is satisfied. In our implementation the cutting plane stops when the increase in the objective function during the last 5 iterations is less than $0.0004 \%$. At the root node this percentage has been fixed to $0.0002 \%$.

\subsection{Upper bounds}

In order to get good upper bounds that decrease the size of the search tree, a heuristic algorithm based on the information given by the fractional solution $x^{*}$ at the end of each node has been implemented.

Since a feasible solution for the WPP will be represented as a directed graph, in what follows we need to distinguish between edges, that will be denoted by $\{i, j\}$, and arcs, denoted by $(i, j)$.

The algorithm first builds a multigraph $G^{*}$ associated with $x^{*}$ in the following way. This graph contains exactly $\left\lfloor x_{i j}^{*}\right\rfloor$ copies of an arc $(i, j)$ when $x_{i j}^{*} \geq 1$, and one copy of arc $(i, j)$ when $0.75 \leq x_{i, j}^{*}<1$. In addition, for each edge $\{i, j\}$ such that $x_{i j}^{*}$ and $x_{j i}^{*}$ are less than 0.75 , we add one arc $(i, j)$ or $(j, i)$ to $G^{*}$. We add the $\operatorname{arc}(i, j)$ if $x_{i j}^{*}>0.5$ and $x_{j i}^{*} \leq 0.5$ or the arc $(j, i)$ if $x_{j i}^{*}>0.5$ and $x_{i j}^{*} \leq 0.5$. Otherwise we add the arc associated with the direction of lower cost.

Consider now the directed graph $G_{a u x}$ resulting from replacing each edge in the original graph $G$ by two opposite arcs. We assign infinite capacity and weights $c_{i j}, c_{j i}$ to these arcs. We add to $G_{\text {aux }}$ one arc $(j, i)$ for each pair of nodes $i, j$ linked in $G^{*}$ by exactly one copy of the arc $(i, j)$ and such that the opposite $\operatorname{arc}(j, i)$ is not in $G^{*}$. These arcs are usually called artificial arcs (since they represent the possibility of reorienting its corresponding opposite arc) and have capacity 2 and weight $\frac{1}{2}\left(c_{j i}-c_{i j}\right)$. Now a minimum cost flow $\left(f_{i j}\right)$ is computed on $G_{a u x}$ with demands and supplies at each vertex defined by the difference between the number of arcs entering to $i$ and leaving from $i$ in $G^{*}$. 
For each non artificial arc $(i, j)$ in $G_{a u x}, f_{i j}$ additional copies of $\operatorname{arc}(i, j)$ are added to $G^{*}$. In addition, for each artificial arc $(j, i)$ with non zero flow we proceed as follows:

- if $f_{j i}=2$ then the $\operatorname{arc}(i, j)$ in $G^{*}$ is replaced by its opposite $\operatorname{arc}(j, i)$.

- if $f_{i j}=1$ then the arc $(i, j)$ in $G^{*}$ is replaced by an edge $\{i, j\}$.

At this point $G^{*}$ can be a mixed graph. We then solve a minimum-cost matching problem defined on a complete graph whose nodes are the ones incident with an odd number of edges in $G^{*}$, and the edge costs are computed from the shortest paths in the original graph as follows. Given two nodes $i$ and $j$, the shortest paths from $i$ to $j$ and from $j$ to $i$ are computed. Then, the average length of each of these two paths is defined as the sum of the average $\operatorname{costs}\left(\frac{c_{u v}+c_{v u}}{2}\right)$ of its corresponding edges $(u, v)$. The minimum of these two lengths is selected as the cost of the edge. Then, for each edge in the optimal matching, we add to $G^{*}$ a copy of each original edge in the corresponding shortest path. Each edge is oriented in the direction given by its smallest cost and another similar flow problem is solved (see also Win 1989). We obtain a directed graph representing a feasible solution for the WPP. This solution is improved by applying three simple procedures described in Benavent et al. (2005). The two first ones look for cycles in the solution graph such that after deleting them or reversing the direction of their arcs, a better solution is obtained. The third one looks for directed paths that can be replaced by a shortest path to obtain a better solution.

\section{Computational experiments}

We present here the computational results obtained on different sets of instances. The $\mathrm{B} \& \mathrm{C}$ procedure has been coded in C/C++ using Cplex 9.1 MIP Solver with Concert Technology 2.0. All the tests were run on an AMD Athlon with Dual Core Processor at $2.41 \mathrm{GHz}$ and $2 \mathrm{~GB}$ RAM with a time limit of 10 hours. In what follows we describe the characteristics of the instances and how they were generated. All the test instances can be found in http://www.uv.es/ corberan/instancias.htm.

\subsection{Data instances}

We have tested the $\mathrm{B} \& \mathrm{C}$ procedure on large WPP and MCPP randomly generated instances that are described next. Type A instances correspond to pure random graphs, while type B instances are associated with graphs that try to imitate street networks.

Three parameters are considered to generate a WPP instance: the number of vertices $n$, $n \in\{500,1000,1500,2000,3000\}$, the vertex degree $d, d \in\{3,4,5,6\}$, and an integer number $a$ used to generate asymmetric costs, $a \in\{10,20,50\}$. First, vertex set $V$ is constructed by randomly generating $n$ points in a square of size $1000 \times 1000$. For the instances of type A, set $E$ is obtained by generating $n \frac{d}{2}$ edges randomly selected as pairs of vertices $(i, j)$. Edge costs are defined as $c_{i j}=\left\lfloor b_{i j}+0.5\right\rfloor$, where $b_{i j}$ denotes the Euclidean distance between $i$ and $j$. If the resulting graph $(V, E)$ is not connected, edges in $d$ different random trees spanning the connected components of the graph are also added to $E$. At this point we have an undirected graph. In order to obtain asymmetric edge costs the first strategy proposed in Win (1987) is applied. First, the value $c_{a}$ is computed as the $a \%$ of the average edge cost $(a \in\{10,20,50\})$. Then, for each edge $e=(i, j) \in E$, let $k_{1}$ and $k_{2}$ be two integer values randomly selected in the interval $\left[-c_{a}, c_{a}\right]$ and set $c_{i j}=\max \left\{1, c_{i j}+k_{1}\right\}$, and $c_{j i}=\max \left\{1, c_{j i}+k_{2}\right\}$. In this way we have generated 60 pure random WPP instances named WA0531 to WA3065, where for example 'WA' 
refers to a WPP instance of type A , ' 05 ' means 500 vertices, ' 3 ' is the vertex degree and ' 1 ' stands for $a=10$.

For the type B instances we proceed as above except in the edges generation step. Here, for each vertex $i \in V$, the $d$ edges connecting $i$ to its $d$ closest neighbors are added to $E$. The idea is to avoid long edges crossing the graph that would not appear in real networks. If the resulting graph is not connected, edges in $d$ different minimal cost spanning trees are also added to $E$. Furthermore, each edge $(i, j) \in E$ such that there is a vertex $k$ satisfying $c_{i j} \geq 0.98\left(c_{i k}+c_{k j}\right)$ is removed from $E$ to avoid 'almost parallel' edges. We have obtained $60 \mathrm{WPP}$ instances of type B named WB0531 to WB3065.

Moreover, 60+60 MCPP instances have been generated in a similar way. The undirected graphs are generated as above, again with $n \in\{500,1000,1500,2000,3000\}$ and $d \in\{3,4,5,6\}$. To obtain mixed graphs each edge is transformed into an arc with probability $p \in\{0.25,0.5,0.75\}$. When a given edge is decided to be changed into an arc, we choose one of its two possible orientations with probability 0.5 . If the resulting graph is not strongly connected, some arcs joining different strongly connected components are changed back into edges. The $120 \mathrm{MCPP}$ instances are named MA0532 (the last '2' refers to $p=0.25$ ) to MB3067.

\subsection{Computational results}

Tables 10 and 11 show the characteristics of the instances above and the computational results obtained on them. They contain the problem type and the number of instances of each set, the number of nodes and the average, minimum and maximum number of edges (and arcs in the case of mixed instances). In both tables the next column shows the number of optimal solutions obtained for each set. For the solved instances (within the time limit of 10 hours), columns "Time" and "B\&C Nodes" present the average computing time (in seconds) and the number of $\mathrm{B} \& \mathrm{C}$ nodes explored. Last column shows the average gap between the final lower bound and the best feasible solution found for those unsolved instances for which a feasible solution was found. In 4 out of 33 unsolved instances (all of them WPPs with 3000 nodes) the algorithm ran out of memory before finishing the study of the root node and no execution of the heuristic algorithm was made.

\begin{tabular}{|c|c|c|c|c|c|c|c|c|c|}
\hline \multirow[b]{2}{*}{ Set } & \multirow{2}{*}{$\begin{array}{c}\text { \# of } \\
\text { Instances }\end{array}$} & \multirow{2}{*}{$\begin{array}{l}\text { \# of } \\
\text { Nodes }\end{array}$} & \multicolumn{3}{|c|}{ \# of Edges } & \multirow{2}{*}{$\begin{array}{c}\text { \# of } \\
\text { Optima }\end{array}$} & \multirow{2}{*}{$\begin{array}{l}\text { Time } \\
\text { (sec.) }\end{array}$} & \multirow{2}{*}{$\begin{array}{r}\text { B\&C } \\
\text { Nodes }\end{array}$} & \multirow{2}{*}{$\begin{array}{l}\text { Gap } \\
(\%)\end{array}$} \\
\hline & & & Aver. & Min & Max & & & & \\
\hline WA05 & 12 & 500 & 1160.5 & 813 & 1518 & 12 & 9.9 & 3.7 & - \\
\hline WB05 & 12 & 500 & 1212.8 & 874 & 1555 & 12 & 14.5 & 0.6 & - \\
\hline WA10 & 12 & 1000 & 2316.8 & 1641 & 3018 & 12 & 168.1 & 27.1 & - \\
\hline WB10 & 12 & 1000 & 2433.9 & 1743 & 3110 & 12 & 174.0 & 2.5 & - \\
\hline WA15 & 12 & 1500 & 3493.1 & 2478 & 4530 & 11 & 188.7 & 75.3 & 0.01 \\
\hline WB15 & 12 & 1500 & 3654.6 & 2631 & 4670 & 12 & 471.8 & 24.3 & - \\
\hline WA20 & 12 & 2000 & 4644.6 & 3303 & 6036 & 12 & 288.0 & 68.7 & - \\
\hline WB20 & 12 & 2000 & 4826.3 & 3467 & 6129 & 8 & 64.6 & 62.7 & 0.12 \\
\hline WA30 & 12 & 3000 & 6961.3 & 4986 & 9066 & 6 & 167.1 & 225.8 & 0.01 \\
\hline WB30 & 12 & 3000 & 7140.8 & 5176 & 9085 & 2 & 3.4 & 110.0 & 0.19 \\
\hline
\end{tabular}

Figure 10: Computational results on WPP instances

As can be seen in Table 10, our algorithm solved all but one of the WPP instances up to 1500 nodes. It was also capable of solving to optimality most of the 2000 nodes WPP instances and some of the 3000 nodes ones. On the other hand, instances of type B (generated trying to 
imitate real networks) proved to be more difficult than those generated completely at random. The results shown in the Table also confirm that our algorithm is capable of solving very large WPP instances with up to 3000 nodes and 9000 edges. Up to our knowledge these are the largest instances ever solved.

\begin{tabular}{|c|c|c|c|c|c|c|c|c|c|c|c|c|}
\hline \multirow[b]{2}{*}{ Set } & \multirow{2}{*}{$\begin{array}{l}\# \text { of } \\
\text { Inst. }\end{array}$} & \multirow{2}{*}{$\begin{array}{c}\text { \# of } \\
\text { Nodes }\end{array}$} & \multicolumn{3}{|c|}{ \# of Edges } & \multicolumn{3}{|c|}{ \# of Arcs } & \multirow{2}{*}{$\begin{array}{l}\text { \# of } \\
\text { Opt. }\end{array}$} & \multirow{2}{*}{$\begin{array}{l}\text { Time } \\
\text { (sec.) }\end{array}$} & \multirow{2}{*}{$\begin{array}{l}\text { B\&C } \\
\text { Nodes }\end{array}$} & \multirow{2}{*}{$\begin{array}{l}\text { Gap } \\
(\%)\end{array}$} \\
\hline & & & Aver. & Min & Max & Aver. & Min & Max & & & & \\
\hline MA05 & 12 & 500 & 615.1 & 351 & 1112 & 542.4 & 193 & 1082 & 12 & 3.2 & 0.7 & - \\
\hline MB05 & 12 & 500 & 626.3 & 320 & 1131 & 583.8 & 202 & 1174 & 12 & 7.5 & 0.4 & - \\
\hline MA10 & 12 & 1000 & 1235.3 & 693 & 2235 & 1083.9 & 340 & 2253 & 12 & 20.0 & 139.8 & - \\
\hline MB10 & 12 & 1000 & 1260.1 & 636 & 2293 & 1182.1 & 416 & 2327 & 11 & 78.6 & 4.0 & 0.01 \\
\hline MA15 & 12 & 1500 & 1851.7 & 1011 & 3423 & 1627.2 & 576 & 3329 & 11 & 83.5 & 38.1 & 0.02 \\
\hline MB15 & 12 & 1500 & 1880.2 & 964 & 3540 & 1750.5 & 615 & 3475 & 12 & 139.9 & 22.8 & - \\
\hline MA20 & 12 & 2000 & 2462.6 & 1355 & 4528 & 2182.8 & 733 & 4515 & 11 & 159.2 & 41.4 & 0.01 \\
\hline MB20 & 12 & 2000 & 2499.8 & 1243 & 4552 & 2329.3 & 850 & 4583 & 10 & 119.6 & 10.6 & 0.18 \\
\hline MA30 & 12 & 3000 & 3704.8 & 1999 & 6795 & 3254.3 & 1097 & 6603 & 8 & 584.7 & 15.9 & 0.09 \\
\hline MB30 & 12 & 3000 & 3746.3 & 1992 & 6799 & 3384.5 & 1206 & 6742 & 9 & 514.0 & 7.0 & 0.97 \\
\hline
\end{tabular}

Figure 11: Computational results on MCPP instances

Table 11 reports the computational results obtained on the MCPP sets of instances. The performance of the $\mathrm{B} \& \mathrm{C}$ procedure is even better on these types of instances with only 12 out of 120 instances remaining unsolved. The algorithm solved to optimality instances with 3000 nodes and up to 9000 arcs and edges, and the average gap obtained on the unsolved instances was less than 1\%. Note that in this case instances of type A and B seem to be of similar difficulty. Another interesting observation is that for a given number of links, instances with a similar number of arcs and edges seem to be harder than those with a bigger proportion of arcs or edges.

\section{Conclusions}

In this paper we have fully described the polyhedra associated with the WPP defined over graphs with up to 4 vertices and 10 edges. Two new families of facet-inducing inequalities have also been presented and, along with the Odd zigzag inequalities, have been proved to be mod-2 cut inequalities. Moreover, a Branch \& Cut algorithm for the exact resolution of the WPP and the MCPP has been described and computational results on several sets of instances have been reported. These results show that the performance of our algorithm is very good, being capable of solving instances of up to 3000 nodes and 9000 links. We think that these results confirm the usefulness of the polyhedral approach to solving difficult combinatorial optimization problems.

Acknowledgments: A. Corberán, I. Plana and J.M. Sanchis wish to thank the Ministerio de Educación y Ciencia of Spain (project MTM2006-14961-C05-02) and the Generalitat Valenciana (project ACOMP06/118) its support.

\section{References}

[1] E. Benavent, A. Carrotta, A. Corberán, J.M. Sanchis \& D. Vigo (2007): "Lower Bounds and Heuristics for the Windy Rural Postman Problem". European Journal of Operational 
Research 176, 855-869.

[2] E. Benavent, A. Corberán, E. Piñana, I. Plana \& J.M. Sanchis (2005): "New Heuristics for the Windy Rural Postman Problem". Computers 86 Operations Research 32, 3111-3128.

[3] P. Brucker, The Chinese Postman Problem for mixed graphs, Proc. Int. Workshop. Lecture Notes in Computer Science 100 (1981), 354-366.

[4] A. Caprara, M. Fischetti \& A.N. Letchford (2000): "On the separation of maximally violated mod- $k$ cuts". Mathematical Programming 87, 37-56.

[5] T. Christof, M. Jünger \& G. Reinelt (1991): "A complete description of the Traveling Salesman Polytope on 8 nodes". Operations Research Letters 10, 497-500.

[6] N. Christofides, E. Benavent, V. Campos, A. Corberán \& E. Mota (1984): "An Optimal Method for the Mixed Postman Problem". In P. Thoft-Christensen (Ed.) System Modelling and Optimization. Lecture Notes in Control and Information Sciences, 59. Berlin: SpringerVerlag.

[7] A. Corberán, I. Plana \& J.M. Sanchis (2005): "The Windy General Routing Polyhedron: A Global View of many Known Arc Routing Polyhedra". Technical Report TR06-2005 (http://www.uv.es/sestio/TechRep/tr06-05.pdf). University of Valencia, Spain. Submitted.

[8] A. Corberán, I. Plana \& J.M. Sanchis (2006), "Zigzag inequalities: A new class of facetinducing inequalities for Arc Routing Problems". Mathematical Programming 108, 79-96.

[9] A. Corberán, I. Plana \& J.M. Sanchis (2007): "A Branch \& Cut Algorithm for the Windy General Routing Problem and special cases". Networks 49, 245-257.

[10] A. Corberán, A. Romero \& J.M. Sanchis (2003): "The Mixed General Routing Problem Polyhedron". Mathematical Programming 96, 103-137.

[11] H.A. Eiselt, M. Gendreau \& G. Laporte (1995): "Arc-Routing Problems, Part 2: the Rural Postman Problem". Operations Research, 43, 399-414.

[12] L.R. Ford \& D.R. Fulkerson (1962): Flows in Networks. Princeton University Press, Princeton, NJ.

[13] L. Fricke \& M. Oswald (2006): Private communication. University of Heidelberg, Germany.

[14] M. Grötschel \& Z. Win (1988): "On the Windy Postman Polyhedron". Report No. 75, Schwerpunktprogram der Deutschen Forschungsgemeinschaft, Universität Augsburg, Germany.

[15] M. Grötschel \& Z. Win (1992): "A Cutting Plane Algorithm for the Windy Postman Problem". Mathematical Programming 55, 339-358.

[16] M. Guan (1984): "On the Windy Postman Problem". Discrete Applied Mathematics, 9, 41-46.

[17] E. Minieka (1979): "The Chinese Postman Problem for Mixed Networks". Management Science 25, 643-648.

[18] D. Naddef \& G. Rinaldi (1991): "The Symmetric Traveling Salesman Polytope and its Graphical Relaxation: Composition of Valid Inequalities". Mathematical Programming 51, $359-400$. 
[19] Y. Nobert \& J.C. Picard (1996): "An Optimal Algorithm for the Mixed Chinese Postman Problem". Networks 27, 95-108.

[20] C.H. Papadimitriou (1976): "On the complexity of edge traversing". Journal of the Association for Computing Machinery 23, 544-554.

[21] T.K. Ralphs (1993): "On the Mixed Chinese Postman Problem". Operations Research Letters 14, 123-127.

[22] K. Wenger (2004): "Generic Cut Generation Methods for Routing Problems". PhD Dissertation, University of Heidelberg, Germany.

[23] Z. Win (1987): "Contributions to Routing Problems". PhD Dissertation, University of Augsburg, Germany.

[24] Z. Win (1989): "On the Windy Postman Problem on Eulerian Graphs". Mathematical Programming 44, 97-112. 